\title{
Muscle activation patterns are bilaterally linked during split-belt treadmill walking in humans
}

M. J. MacLellan, Y. P. Ivanenko, F. Massaad, S. M. Bruijn, J. Duysens and F. Lacquaniti

J Neurophysiol 111:1541-1552, 2014. First published 29 January 2014; doi:10.1152/jn.00437.2013

You might find this additional info useful...

This article cites 42 articles, 16 of which can be accessed free at:

/content/111/8/1541.full.html\#ref-list-1

This article has been cited by 1 other HighWire hosted articles

Modulation of phase durations, phase variations, and temporal coordination of the four

limbs during quadrupedal split-belt locomotion in intact adult cats

Giuseppe D'Angelo, Yann Thibaudier, Alessandro Telonio, Marie-France Hurteau, Victoria

Kuczynski, Charline Dambreville and Alain Frigon

J Neurophysiol, October 15, 2014; 112 (8): 1825-1837.

[Abstract] [Full Text] [PDF]

Updated information and services including high resolution figures, can be found at:

/content/111/8/1541.full.html

Additional material and information about Journal of Neurophysiology can be found at: http://www.the-aps.org/publications/jn

This information is current as of December 3, 2014. 


\title{
Muscle activation patterns are bilaterally linked during split-belt treadmill
}

\author{
walking in humans
}

\author{
M. J. MacLellan, ${ }^{1,2}$ Y. P. Ivanenko, ${ }^{2}$ F. Massaad, ${ }^{3}$ S. M. Bruijn, ${ }^{3,4}$ J. Duysens, ${ }^{3,5}$ \\ and F. Lacquaniti ${ }^{2,6,7}$ \\ ${ }^{1}$ School of Kinesiology, Louisiana State University, Baton Rouge, Louisiana; ${ }^{2}$ Laboratory of Neuromotor Physiology, IRCCS Santa \\ Lucia Foundation, Rome, Italy; ${ }^{3}$ Motor Control Laboratory, Research Center for Movement Control and Neuroplasticity, \\ Department of Biomedical Kinesiology, Katholieke Universiteit Leuven, Leuven, Belgium; ${ }^{4}$ Department of Orthopedics, First \\ Affiliated Hospital of Fujian Medical University, Fuzhou, Fujian, People's Republic of China; ${ }^{5}$ Department of Research, \\ Development and Education, Sint Maartenskliniek, Nijmegen, The Netherlands; ${ }^{6}$ Centre of Space Bio-medicine, University of Rome \\ Tor Vergata, Rome, Italy; and ${ }^{7}$ Department of Systems Medicine, University of Rome Tor Vergata, Rome, Italy
}

Submitted 17 June 2013; accepted in final form 24 January 2014

\begin{abstract}
MacLellan MJ, Ivanenko YP, Massaad F, Bruijn SM, Duysens J, Lacquaniti F. Muscle activation patterns are bilaterally linked during split-belt treadmill walking in humans. J Neurophysiol 111: 1541-1552, 2014. First published January 29, 2014; doi:10.1152/jn.00437.2013.There is growing evidence that human locomotion is controlled by flexibly combining a set of basic muscle activity patterns. To explore how these patterns are modified to cope with environmental constraints, 10 healthy young adults 1 st walked on a split-belt treadmill at symmetric speeds of 4 and $6 \mathrm{~km} / \mathrm{h}$ for $2 \mathrm{~min}$. An asymmetric condition was then performed for $10 \mathrm{~min}$ in which treadmill speeds for the dominant (fast) and nondominant (slow) sides were 6 and $4 \mathrm{~km} / \mathrm{h}$, respectively. This was immediately followed by a symmetric speed condition of $4 \mathrm{~km} / \mathrm{h}$ for $5 \mathrm{~min}$. Gait kinematics and ground reaction forces were recorded. Electromyography (EMG) was collected from 12 lower limb muscles on each side of the body. Nonnegative matrix factorization was applied to the EMG signals bilaterally and unilaterally to obtain basic activation patterns. A cross-correlation analysis was then used to quantify temporal changes in the activation patterns. During the early (1st 10 strides) and late (final 10 strides) phases of the asymmetric condition, the patterns related to ankle plantar flexor (push-off) of the fast limb and quadriceps muscle (contralateral heel contact) of the slow limb occurred earlier in the gait cycle compared with the symmetric conditions. Moreover, a bilateral temporal alignment of basic patterns between limbs was still maintained in the split-belt condition since a similar shift was observed in the unilateral patterns. The results suggest that the temporal structure of these locomotor patterns is shaped by sensory feedback and that the patterns are bilaterally linked.
\end{abstract}

asymmetric gait; motor control; sensory feedback

LOCOMOTOR BEHAVIORS INVOLVE the activation of many muscles. There is a large body of work that suggests animal locomotion is controlled by spinal central pattern generators (CPGs; Grillner 1979; Kiehn 2006). It has also been proposed that the mammalian CPG has two layers: a rhythm-generating layer distinct from a pattern-generating layer (McCrea and Rybak 2008; Patla 1985). Such organization provides flexibility and the ability to adapt gait patterns to environmental demands. However, definitive evidence does not currently exist linking the results from animal studies to human locomotion. Although quasiautomatic adaptive changes occur frequently in our daily

Address for reprint requests and other correspondence: M. J. MacLellan, School of Kinesiology, Louisiana State Univ., Baton Rouge, LA 70803 (e-mail: michaelm@1su.edu). lives, we do not have a complete understanding as to how the central nervous system (CNS) accomplishes these tasks. Despite an involvement of numerous muscles, evidence in the literature also suggests that human locomotion may be controlled by flexibly combining a small set of muscle activity patterns (Lacquaniti et al. 2012). In particular, an essential feature of the controller is a tight bilateral coordination of the lower limbs and their muscle activations (Ivanenko et al. 2006; Olree and Vaughan 1995).

Various techniques have been used to quantify the motor output that arises from this neural control. One such technique has used factorization algorithms (d'Avella et al. 2003; Giszter and Hart 2013; Ting et al. 2012; Tresch et al. 2006) and shown that a large proportion of the variance in muscle activation during locomotion can be accounted for by $4-5$ basic patterns (Davis and Vaughan 1993; Ivanenko et al. 2004; Neptune et al. 2009). It has been hypothesized that these basic activation patterns extracted from electromyographic (EMG) data may be representative of the previously mentioned CPG output (Dominici et al. 2011; Ivanenko et al. 2003, 2004, 2005; Lacquaniti et al. 2012).

There is a debate as to whether the two lower limbs are controlled together or independently by neural networks. Evidence in infants (Yang et al. 2005) and paradigms that examine transfer of learning between limbs (Choi and Bastian 2007) have suggested independent neural control for right and left lower limbs. On the other hand, anatomic and electrophysiological studies have identified commissural interneurons that may be involved in coordinating locomotion between the two sides in the spinal cord of animal models (Butt and Kiehn 2003). Also, past work using factor analysis on muscle activity recordings has identified bilateral common factors, which are aligned with a half-gait cycle shift, therefore coordinating right and left sides (Ivanenko et al. 2006; Olree and Vaughan 1995). One way to address the issue of coordination between the lower limbs is to elicit asymmetric walking such as on a split-belt treadmill.

The use of a split-belt treadmill provides a novel environment in which limb coordination must be adjusted to maintain stability and forward progression. Adaptations to split-belt locomotion were initially studied in cats (Forssberg et al. 1980; Halbertsma 1983) and subsequently in healthy human subjects (Choi and Bastian 2007; Dietz et al. 1994; Prokop et al. 1995; 
Reisman et al. 2005), infants (Thelen et al. 1987; Yang et al. 2005), as well as pathological populations (Morton and Bastian 2006; Nanhoe-Mahabier et al. 2013; Reisman et al. 2007). In these studies, there is an adjustment of limb kinematic timing such that a decrease of stance time and an increase of swing time in the faster moving limb occurs in conjunction with an increase of stance time and a decrease in swing time in the slower moving limb. The result from these adjustments is a maintenance of the 1:1 stepping relationship between limbs. During a bout of split-belt walking, there is a fine-tuning of interlimb coordination throughout, and aftereffects are seen postadaptation (Reisman et al. 2005). This fine-tuning of interlimb coordination is accompanied by a reduction in muscle activity that may be related to increasing the economy of energy expenditure throughout adaptation (Finley et al. 2013). It is thought that these kinematic changes are related in part to proprioceptive information from the lower limb (including position, velocity, and loading), which influences the motor output (Dietz et al. 1994; Prokop et al. 1995). If the resulting motor output is related to a combination of basic activity patterns, how do these patterns respond to the above-mentioned proprioceptive feedback and additional supraspinal influences associated with walking on a split-belt treadmill?

The present study used a split-belt paradigm to determine what temporal changes, if any, occur to locomotor activity patterns. One possibility (hypothesis 1) is that these changes are correlated bilaterally (Ivanenko et al. 2006; Olree and Vaughan 1995), perhaps also due to the strong commissural interneuron linkages between the left and right sides (Butt and Kiehn 2003), so that similar temporal changes in activity patterns may be expected between the two sides. It is also worth stressing that, despite an asymmetric kinematic behavior, a 1:1 relationship between limbs remains in a split-belt condition so that the cycle durations are the same for the right and left legs. This may facilitate temporal synchronization of basic activation patterns on the two sides of the body. Alternatively (hypothesis 2), because it has been suggested that each limb can be controlled independently (Choi and Bastian 2007; Yang et al. 2005), temporal shifts of activity patterns may occur independently of each other. The ability to discriminate between these two hypotheses may provide insights into the debate between independent and interdependent control of limb pattern generators.

\section{METHODS}

Participants. Ten healthy young adults [2 women, 8 men, age: mean 22.3 (SD 3.1) yr, weight: mean $70.7 \pm 11.4 \mathrm{~kg}$, height: $1.77 \pm$ $0.10 \mathrm{~m}, 2$ left-leg dominant, 8 right-leg dominant] participated in the study. The studies were in accordance with the Declaration of Helsinki, and informed, written consent was obtained from all participants according to procedures approved by the Biomedical Research Ethics Committee at the Katholieke Universiteit (KU) Leuven (Leuven, Belgium).

Protocol. Participants walked on a split-belt treadmill (ForceLink, Culemborg, The Netherlands) for a series of speed conditions. Initially, tied-belt conditions (both belts moving at the same speed for 2 min) were performed at 4 or $6 \mathrm{~km} / \mathrm{h}$, these speeds being randomly assorted between trials (Fig. 1A). Following the baseline condition, a split-belt condition was performed for $10 \mathrm{~min}$, whereby the treadmill belt speed was $6 \mathrm{~km} / \mathrm{h}$ for the dominant limb and $4 \mathrm{~km} / \mathrm{h}$ for the nondominant limb $(6: 4 \mathrm{~km} / \mathrm{h})$. Limb dominance was determined using the Waterloo Footedness Questionnaire-Revised (Elias et al. 1998) and a clinical test (Schneiders et al. 2010). We refer to the dominant and nondominant limbs as "fast" and "slow" limbs, respectively. Immediately after this split-belt condition, a tied-belt condition (postasymmetry) was performed for 5 min with both belts moving at 4 $\mathrm{km} / \mathrm{h}$. During the time between split-belt and postasymmetry conditions $(\sim 30 \mathrm{~s})$, participants stood unsupported on the treadmill with their eyes open. From all conditions, specific recording windows were selected for all analyses of data: final 10 strides from the 4 and $6 \mathrm{~km} / \mathrm{h}$ baseline conditions (referred to as tied $4 \mathrm{~km} / \mathrm{h}$ and tied $6 \mathrm{~km} / \mathrm{h}$ ), $1 \mathrm{st} 10$ strides and last 10 strides from the $6: 4 \mathrm{~km} / \mathrm{h}$ condition (referred to as early and late split-belt), and 1 st 10 strides and last 10 strides from the $4 \mathrm{~km} / \mathrm{h}$ condition following the $6: 4 \mathrm{~km} / \mathrm{h}$ condition (referred to as early and late postasymmetry). Ten strides were selected as a compromise between five strides previously used to determine split-belt adaptations (Reisman et al. 2005) while allowing for appropriate averaging of muscle activity waveforms (Ivanenko et al. 2004). Means over the 10 strides for each independent variable were calculated for each participant.

Data recording and analysis. Full-body, 3-dimensional kinematics were recorded using a 10-camera Vicon MX system (Oxford Metrics, Oxford, United Kingdom) at 100 samples per second. Reflective markers were placed on the body according to the full-body, plug-ingait model provided in the Vicon system software. All kinematic data were low-pass filtered offline with a 2 nd-order, dual-pass (0-phase) Butterworth filter with a cutoff frequency of $7 \mathrm{~Hz}$. In addition, ground reaction forces were recorded by means of force plates integrated into the treadmill at a frequency of 2,000 samples per second. These data were low-pass filtered offline using a 2nd-order, dual-pass (0-phase) Butterworth filter with a cutoff frequency of $50 \mathrm{~Hz}$.

Twenty-four channels of surface EMG were collected at 2,000 samples per second using a ZeroWire system (Aurion, Milan, Italy). The muscles recorded bilaterally in the current study were: gluteus maximus (GM), tensor fasciae latae (TFL), adductor magnus (ADD), rectus femoris (RF), vastus lateralis (VL), vastus medialis (VM), semitendinosus (ST), biceps femoris (BF), lateral gastrocnemius (LG), medial gastrocnemius (MG), soleus (SOL), and tibialis anterior (TA). Electrode placement locations were determined using SENIAM (Surface ElectroMyoGraphy for the Non-Invasive Assessment of Muscles) guidelines (Hermens et al. 2000). All EMG data were initially high-pass filtered (30-Hz cutoff) offline using a 2nd-order, dual-pass Butterworth filter and band-stop filtered at a frequency of 50 Hz. EMG data were then full-wave rectified and low-pass filtered using a 2nd-order, dual-pass (0-phase) Butterworth filter with a cutoff of $10 \mathrm{~Hz}$. Finally, EMG data were time-interpolated to 200 points over a gait cycle for interstride averaging.

Force-plate data were used to determine the times of heel contact (when the force in the vertical direction exceeded $15 \mathrm{~N}$ ) and toe lift-off (when the force in the vertical direction decreased below 15 $\mathrm{N}$ ). For all analysis and figures, the gait cycle begins with heel contact of the fast-moving limb. From these data, individual stride and swing times were calculated. The kinematic data were used to calculate limb excursion in each limb (the horizontal distance traveled by the heel marker from initial contact to lift-off), and, from these data, limb excursion difference was calculated as the limb excursion of the fast limb subtracted from the slow limb [using the terminology suggested in Hoogkamer et al. (2014)]. To quantify EMG amplitude throughout the stride, muscle activation patterns were integrated over a stride cycle (iEMG) for all muscles recorded. Before integration, each muscle was normalized in amplitude to the maximum amplitude over all conditions. The iEMG ratios for each muscle were calculated for the split-belt and postasymmetry conditions by dividing the late iEMG by the early iEMG as shown in Finley et al. (2013). A ratio of $<1$ would indicate the iEMG decreased from the early to late stages of the condition.

Basic activation patterns. Nonnegative matrix factorization (NNMF) was used to identify underlying patterns in the EMG recordings using a linear decomposition according to the following formula: 
A

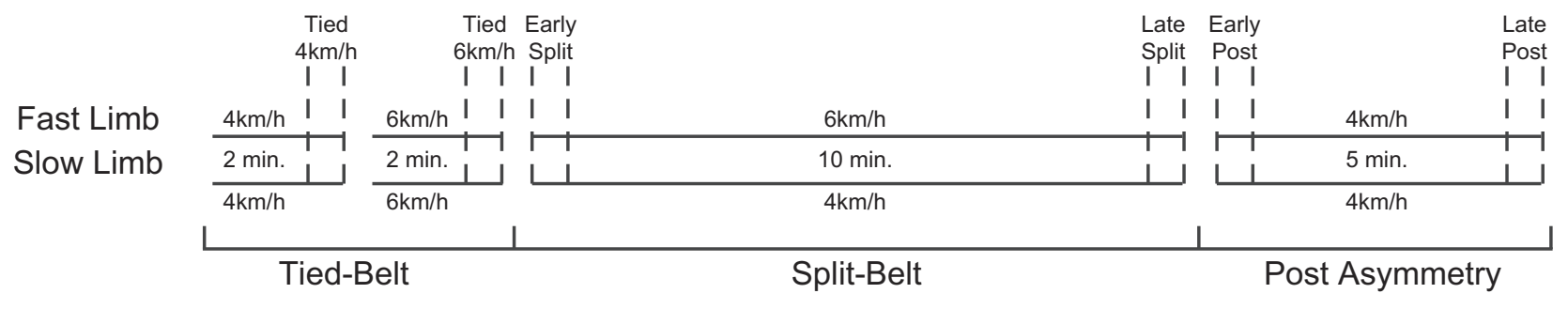
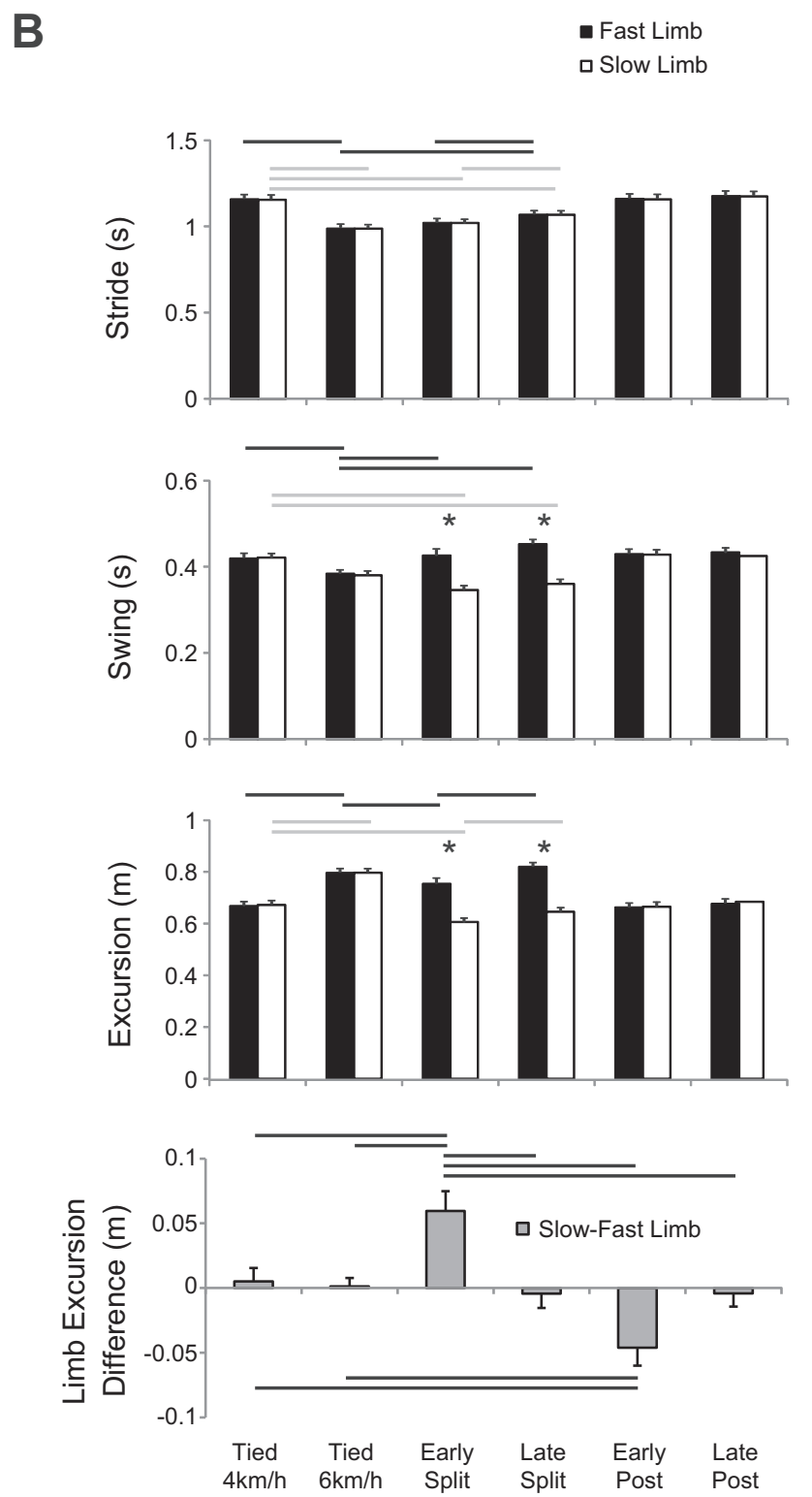

Fig. 1. Experimental protocol and kinematic gait characteristics. Recordings of 10 strides were made at specific instances (represented by dashed lines) during the protocol and are illustrated in $A$. Group-averaged kinematic gait characteristics ( \pm SE) for the fast (black) and slow (white) limbs are displayed in $B$. Statistical differences $(P<0.05)$ between conditions are shown with solid and dashed lines for fast and slow limbs, respectively. * Significant differences $(P<0.05)$ between limbs.

$$
E=W \cdot C+\text { residual, }
$$

where $E$ ( $m \times t$ matrix, where $m$ is the number of muscles and $t=200$ samples, with the spatiotemporal profiles of muscle activity) is assumed be a linear combination of weighting coefficients (or loadings),
$W$ ( $m \times n$ matrix, where $n$ is the number of patterns), and basic activation patterns (or temporal components), $C(n \times t$ matrix). The analysis was performed in 3 different ways: bilaterally (24 EMG recordings from left and right side, time normalized to fast limb heel contact), unilateral fast limb, and unilateral slow limb (12 EMG 
recordings from each side, time normalized to heel contact of the fast limb).

Before NNMF, individual EMG recordings for each muscle were averaged over the 10 strides for the window being analyzed and were subsequently normalized in magnitude. This normalization consisted of dividing each data point for a specific muscle by the maximum amplitude of that muscle over all averaged windows. This procedure was performed for each participant individually. If an EMG signal appeared to contain artifacts, this muscle was deleted for the particular stride (this occurred very infrequently). To determine the appropriate number of activity patterns from the analysis, NNMF was applied using 1-8 patterns ( $C$ in $E q .1$ ), and the percentage of variance (PoV) accounted for by these patterns was determined and ordered for illustration purposes with respect to pattern peak timing. The results were then averaged over the 10 participants for each individual condition, and if the addition of a pattern did not explain $>7 \%$ of the variance, the remaining ones were not taken into account. This cutoff value generally corresponded with the sharp change in slope seen in the PoV plots that has been used previously to determine the number of patterns to be retained (d'Avella et al. 2003). Our procedure resulted in retaining four patterns for both the bilateral and unilateral analyses. On average, the PoV accounted for by these patterns was 82.5, 91.8, and $91.2 \%$ for the bilateral, unilateral fast limb, and unilateral slow limb analyses, respectively. For illustration purposes, each pattern (and the corresponding loading) was normalized to the peak magnitude of the pattern. To quantify changes in the temporal position of each pattern, a cross-correlation was performed to determine the phase difference (presented as a percentage change in the gait cycle) using the tied-belt $4 \mathrm{~km} / \mathrm{h}$ condition as a reference.

Statistics. To determine statistical differences between conditions, a one-way repeated-measures ANOVA was applied to all kinematic data. If this ANOVA was significant for the kinematic variables, planned comparisons were performed using a Tukey test. For both limbs, planned comparisons were performed between 4 and $6 \mathrm{~km} / \mathrm{h}$, early and late split-belt, early and late postasymmetry, $4 \mathrm{~km} / \mathrm{h}$ and early postasymmetry, and $4 \mathrm{~km} / \mathrm{h}$ and late postasymmetry. However,

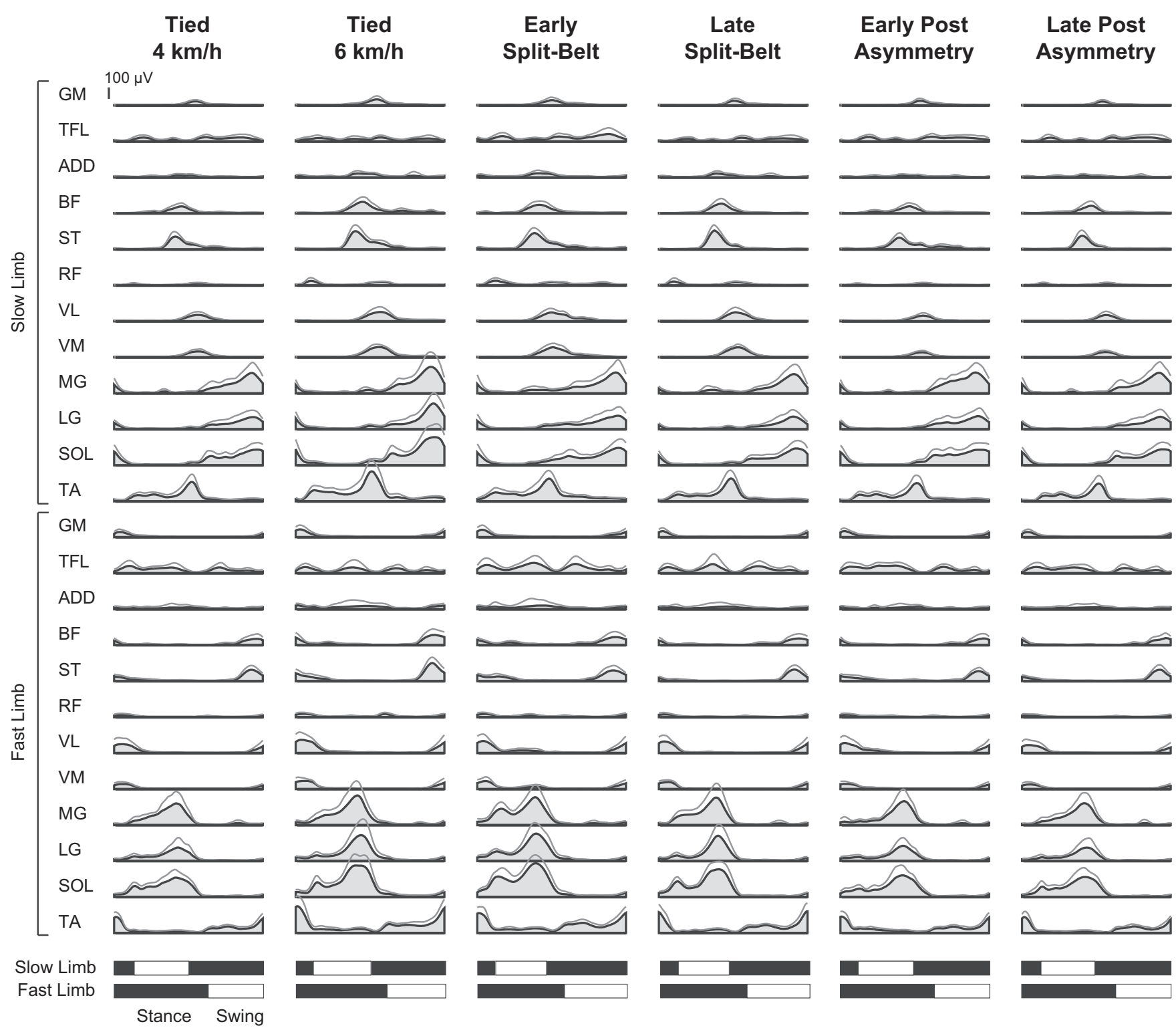

Fig. 2. Muscle activity patterns in each limb for each locomotor condition. Group-averaged muscle activity patterns (gray area with black outline) and SD (gray lines) are illustrated for the gluteus maximus (GM), tensor fascia latae (TFL), adductor magnus (ADD), biceps femoris (BF), semitendinosus (ST), rectus femoris (RF), vastus lateralis (VL), vastus medialis (VM), medial gastrocnemius (MG), lateral gastrocnemius (LG), soleus (SOL), and tibialis anterior (TA). Muscle activations are displayed during the fast limb stride cycle (seen on bottom). 
for the individual limbs in the split-belt conditions, the planned comparisons were dependent on the limb being examined; the treadmill speed for the fast limb in the split-belt condition was $6 \mathrm{~km} / \mathrm{h}$, and therefore comparisons for this limb were performed with the tied-belt speed of $6 \mathrm{~km} / \mathrm{h}(6 \mathrm{~km} / \mathrm{h}$ and early split-belt and $6 \mathrm{~km} / \mathrm{h}$ and late split-belt). Similar comparisons were made for the slow limb with a belt speed of $4 \mathrm{~km} / \mathrm{h}(4 \mathrm{~km} / \mathrm{h}$ and split-belt and $4 \mathrm{~km} / \mathrm{h}$ and late split-belt). A single-sample $t$-test was used to determine whether changes in iEMG occurred between early and late stages of the split-belt and postasymmetry conditions. In addition, a one-way repeated-measures ANOVA was applied to the output of the crosscorrelation analysis applied to the patterns extracted from EMG data, and a Tukey test was used to make post hoc comparisons. Statistical significance was set at $P<0.05$.

\section{RESULTS}

Gait kinematics. Consistent with previous results, temporal aspects of gait kinematics were adjusted when walking on the split-belt treadmill (Fig. $1 B$ ). In the current study, stride time changed in the fast $\left[F_{(5,40)}=67.62, P<0.001\right]$ and slow $\left[F_{(5,40)}=60.41, P<0.001\right]$ limbs over the different treadmill speeds; however, these times are similar between the slow and fast limbs. In each limb, stride time was lower in the 6 than the $4 \mathrm{~km} / \mathrm{h}$ condition $(P<0.001)$ as well as in the early compared with late split-belt conditions (fast: $P<0.05$, slow: $P<0.05$ ). Fast limb stride time was also greater in the late split-belt condition compared with the tied-belt $6 \mathrm{~km} / \mathrm{h}$ condition $(P<$ $0.001)$. Slow limb stride time was lower for early $(P<0.001)$ and late $(P<0.001)$ split-belt conditions compared with the 4 $\mathrm{km} / \mathrm{h}$ tied-belt condition.

These changes in stride time were reflected in swing time modulations [fast: $F_{(5,40)}=10.36, P<0.001$; slow: $F_{(5,40)}=$ 91.53, $P<0.001$; Fig. $1 B]$. Again, swing time was lower in the $6 \mathrm{~km} / \mathrm{h}$ compared with the $4 \mathrm{~km} / \mathrm{h}$ condition for the fast $(P<$ $0.01)$ and slow $(P<0.001)$ limbs. In the fast limb, swing time was longer in the early $(P<0.01)$ and late $(P<0.001)$ split-belt conditions compared with the equivalent tied-belt speed. A decrease in swing time was shown for the early $(P<$ $0.001)$ and late $(P<0.001)$ split-belt conditions compared with the $4 \mathrm{~km} / \mathrm{h}$ tied-belt speed in the slow limb. Additionally, swing time was shown to increase in the slow limb between early and late split-belt conditions $(P<0.05)$. Between limbs, swing time was greater in the fast limb for the early $(P<$ $0.001)$ and late $(P<0.001)$ split-belt conditions.

In addition, limb excursion difference (an interlimb variable) showed changes throughout the split-belt conditions. A main effect was shown across belt speed conditions $\left[F_{(5,40)}=21.01\right.$, $P<0.001]$, and further analysis suggested that the limb excursion difference was greater in the early split-belt condition compared with all other belt conditions $(P<0.01)$, and this difference was lower in the early postasymmetry condition compared with the tied-belt conditions $(P<0.05)$.

In sum, these kinematic variables displayed similar patterns to those seen in previous work examining temporal and spatial changes to split-belt locomotion (Dietz et al. 1994; Prokop et al. 1995; Reisman et al. 2005).

Gait EMG. Figure 2 illustrates EMG patterns for all recorded muscles during the examined treadmill speed conditions. With visual inspection, one can see that the EMG waveforms are similar in shape throughout all conditions, although the amplitudes of these waveforms can change. For instance, analysis of iEMG (Fig. 3) suggested that most muscles (21 of 24) decreased in amplitude between the early and late stages of the split-belt condition. To a lesser extent, the iEMG amplitude of 10 of the 24 muscles decreased between the early and late stages of the postasymmetry condition.

Figure 4 shows the results obtained by pooling together the EMG data from both left and right lower limbs (bilateral analysis). The four activity patterns selected from the analysis are consistent across all participants (as seen by the extent of superposition of the traces obtained in each individual). The labels of these patterns used in the following description are with reference to the location of the pattern peak with reference to fast limb contact (for example, the pattern containing the 1st peak that occurs after fast limb contact is called pattern 1). Examination of the pattern loadings shows which muscles are related to each of the patterns. Patterns 1 and 3 are related to ipsilateral hip (GM) and knee (RF, VL, and VM) extensor activity for the fast (pattern 1) and slow (pattern 3) limbs. Patterns 2 and 4 are related to ipsilateral plantar flexor activity (MG, LG, and SOL) and contralateral hamstring activity (BF
Fast Limb
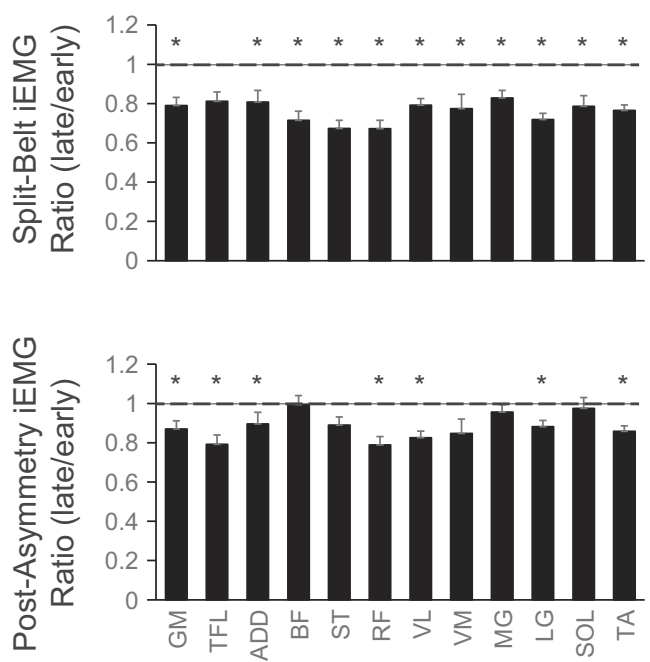

Slow Limb
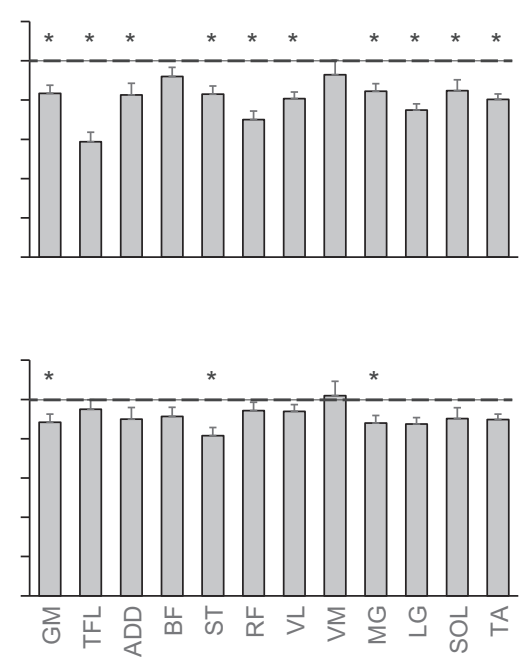

Fig. 3. Changes in integrated electromyographic (iEMG) magnitude during split-belt and postasymmetry conditions. Ratios of iEMG (late/early) are illustrated for the fast (left column) and slow (right column) limbs during the split-belt (top row) and postasymmetry (bottom row) conditions. Each bar represents the group mean, and the SE is shown. A value of 1 (shown with a dashed line) would indicate no change in IEMG magnitude between the early and late stages of the condition. *iEMG ratios significantly different from $1(P<0.05)$. 


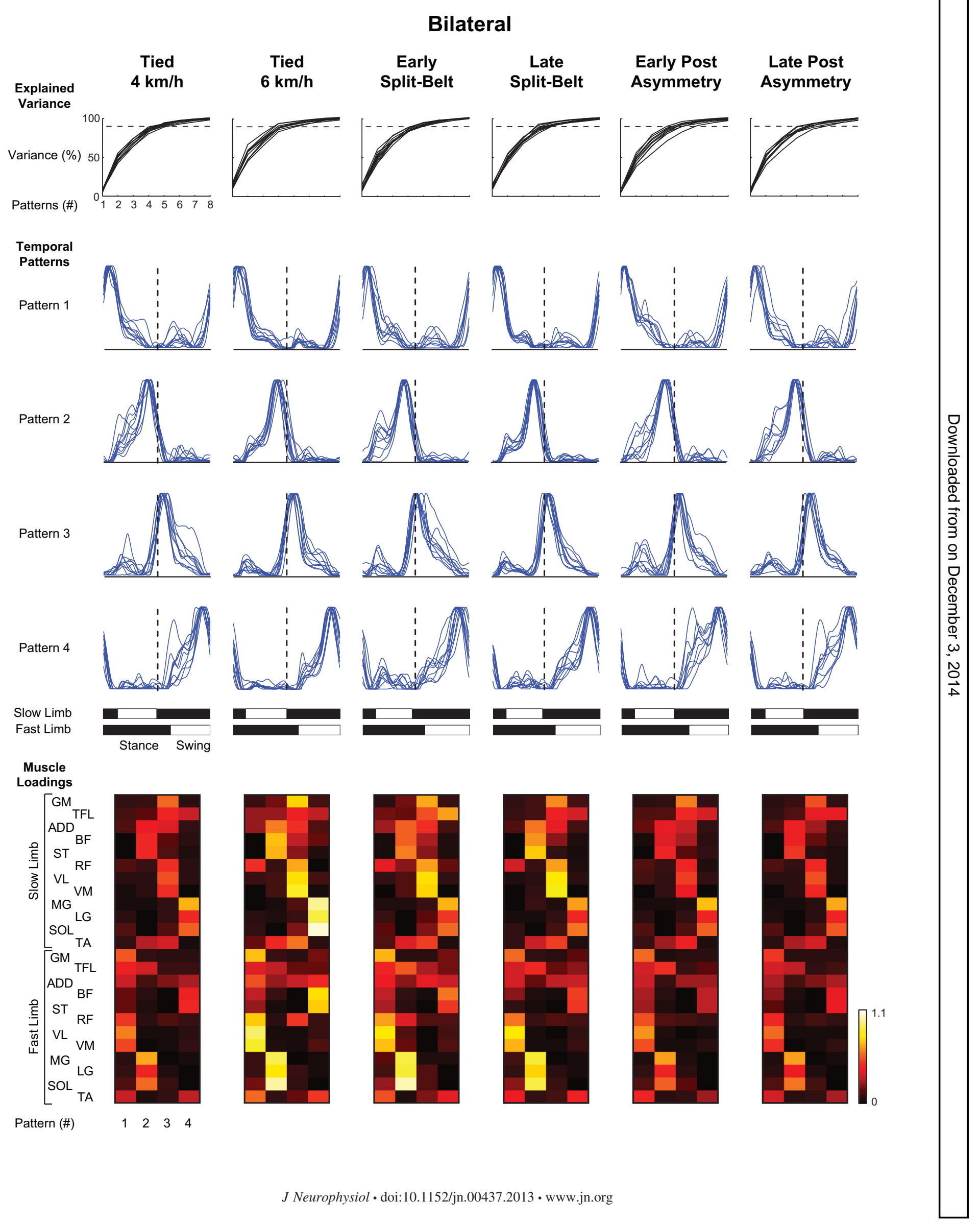


and ST) in the fast (pattern 2) and slow (pattern 4) limbs. Statistical analysis suggested that the timing of the first and fourth patterns remained consistent throughout all experimental conditions (main effects across treadmill speed conditions in patterns 1 and 4 revealed $P>0.05$; Fig. 7A). However, temporal changes were observed in the second $\left[F_{(4,32)}=20.49\right.$, $P<0.001]$ and third $\left[F_{(4,32)}=9.04, P<0.001\right]$ patterns. The second pattern occurred earlier in the gait cycle during the early $(P<0.001)$ and late $(P<0.05)$ split-belt conditions compared with all tied-belt conditions. Also, the third pattern displayed a similar shift and occurred earlier in the gait cycle for the early $(P<0.001)$ and late $(P<0.05)$ compared with the tied-belt conditions.

NNMF analyses for the fast- and slow-moving limbs are shown in Figs. 5 and 6, respectively. In each of these limbs, the shapes of three of these patterns were relatively similar across participants (as seen in the bilateral analysis), whereas the fourth pattern was somewhat less consistent. Only the three consistent patterns were retained for statistical analysis. The loadings of these patterns were similar between limbs with one pattern being related to hip (GM) and knee (RF, VL, and $\mathrm{VM}$ ) extensor muscles, a second related to ankle plantar flexors (LG, MG, and SOL), and a third one to knee flexor/hip extensor (BF and ST) activity. In the fast limb, a change in the temporal position (Fig. 7A) only occurred in the second pattern $\left[F_{(4,32)}=22.36, P<0.001\right]$. Post hoc analysis suggested that the peak of this pattern occurred earlier in the early $(P<$ $0.001)$ and late $(P<0.001)$ split-belt treadmill condition compared with all tied-belt conditions. Moreover, the slow limb showed a significant main effect for the second $\left[F_{(4,32)}=\right.$ 12.07, $P<0.001]$ and third $\left[F_{(4,32)}=4.50, P<0.005\right]$ patterns. Further analysis showed that the second pattern occurred earlier in the early split-belt compared with the $6 \mathrm{~km} / \mathrm{h}$ tied-belt condition $(P<0.005)$. Also, this second pattern occurred earlier in the late split-belt condition compared with all tied conditions $(P<0.005)$. Post hoc analysis suggested that the third pattern occurred earlier in the early split-belt condition compared with all tied-belt conditions $(P<0.05)$.

To explore the kinematic events with which these temporal shifts were associated, the toe-off time of the fast limb and the contact time of the slow limb with respect to the fast gait cycle were examined (Fig. $7 B$ ). We found significant changes in fast limb toe-off $\left[F_{(4,32)}=36.68, P<0.001\right]$; further analysis showed that toe-off occurred before in the early $(P<0.001)$ and late $(P<0.001)$ split-belt conditions compared with all tied-belt conditions. In addition, toe-off occurred before in the $6 \mathrm{~km} / \mathrm{h}$ tied condition compared with early $(P<0.05)$ and late $(P<0.05)$ postasymmetry. A main effect was shown for the slow limb contact $\left[F_{(4,32)}=44.04, P<0.001\right]$, and, similar to pattern timings, this contact time occurred earlier in the gait cycle for the early $(P<0.001)$ and late $(P<0.001)$ split-belt compared with the tied-belt conditions. Moreover, the slow limb contact occurred before in the early postcondition compared with the tied $6 \mathrm{~km} / \mathrm{h}$ condition $(P<0.05)$.

\section{DISCUSSION}

Locomotion on a split-belt treadmill leads to asymmetric changes in lower limb movement. Our findings support previous work showing a modulation of swing time in each limb during the split-belt locomotor conditions (Dietz et al. 1994; Prokop et al. 1995; Reisman et al. 2005). The novel aspect of the current study is the analysis of multimuscle EMG patterns during split-belt treadmill locomotion and the application of NNMF to show the adjustments of the temporal structure of the activation patterns. The adjustments included the maintenance of some patterns (e.g., patterns 1 and 4, Fig. 7A, left) and a temporal shift to an earlier time in the gait cycle in the others (patterns 2 and 3, Fig. 7A, left). Despite these temporal shifts, a bilateral alignment between limbs was still present since a similar shift occurred in the unilateral patterns (Fig. 7A), consistent with hypothesis 1 on parallel changes in basic activation patterns between the two sides. Below, we discuss the related kinematic and EMG modifications investigated in this study.

Changes in kinematic and individual muscle activity patterns. Kinematic modifications to split-belt treadmill locomotion have been examined in human adults (Choi and Bastian 2007; Dietz et al. 1994; Prokop et al. 1995; Reisman et al. 2005), infants (Thelen et al. 1987; Yang et al. 2005), and cats (Forssberg et al. 1980). This previous work provided converging evidence for the modulation of stance and swing times, whereby a decrease in stance time and an increase in swing time is present in the faster moving limb, with opposite effects seen in the slower moving limb. In addition, a refinement of these kinematic patterns occurs throughout a bout of split-belt walking. For instance, Reisman et al. (2005) showed a steady increase of step length for the fast limb, ultimately reaching a plateau by the end of a split-belt walking condition, when step length is equal again between fast and slow limbs, suggesting an adaptation throughout the trial. The results in this study show changes of limb excursion difference between early and late split-belt locomotion (Fig. 1B) as well as a posteffect in the early postadaptation condition, similar to the adjustments seen in step length difference in Reisman et al. (2005). These adaptations seen during split-belt gait have led previous authors to conclude that feedback from lower limb position, velocity, and loading affect locomotor output (Dietz et al. 1994; Prokop et al. 1995), consistent with a general contribution of proprioceptive feedback during walking.

Less attention in the literature has been directed to EMG activity during split-belt locomotion. Using a smaller set of muscles, Dietz et al. (1994) showed an increase in gastrocnemius activity and decrease in tibialis anterior activity on the faster and slower moving sides, respectively, consistent with more recent

Fig. 4. Nonnegative matrix factorization of bilateral muscle activations. The top row of line plots illustrates the percentage of variance explained by decomposing the EMG signals into 1-8 patterns. Each black line in these plots represents the percentage of explained variance calculated for a single participant. The horizontal dashed line in these plots indicates $90 \%$ of explained variance. The middle portion of the figure shows the 4 temporal patterns extracted from the EMG data that are ordered by location of the dominant peak with reference to heel contact of the fast limb. Each blue trajectory shows a temporal pattern extracted for a single participant, and the dashed vertical line indicates 50\% of the fast limb gait cycle. Stance and swing phases for the fast and slow limbs are shown below for reference. Group-averaged pattern loadings for the 4 extracted patterns are shown in the bottom portion of the figure. Note the asymmetry between fast and slow limbs for the loadings related to the quadriceps (RF, VL, and VM) and ankle plantar flexor (MG, LG, and SOL) muscles. 


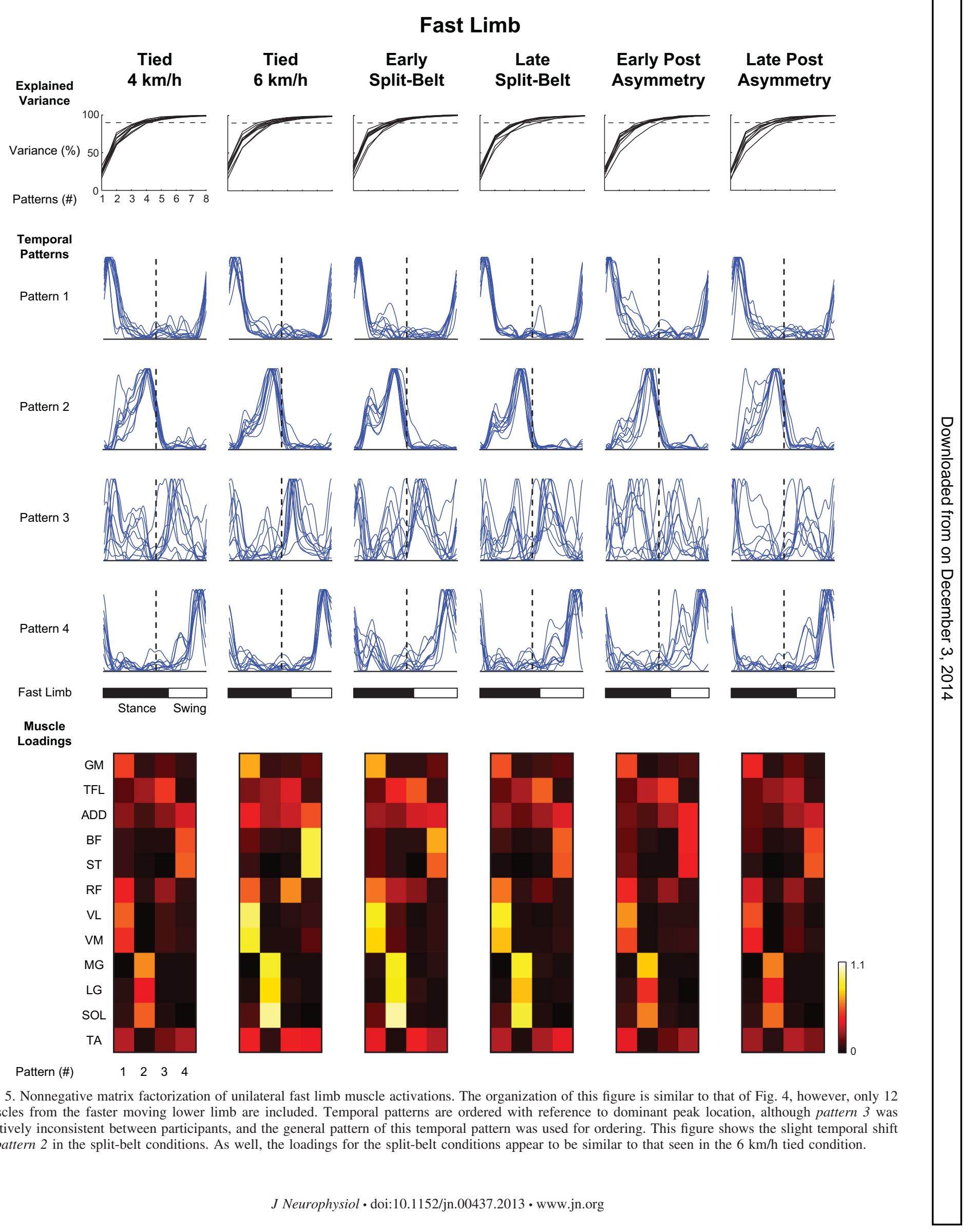




\section{Slow Limb}

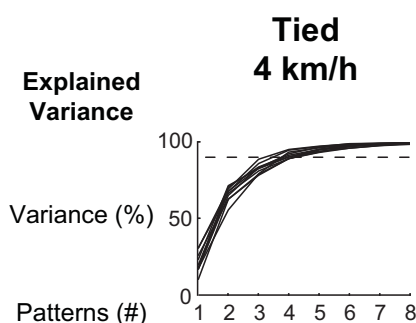

Tied $6 \mathrm{~km} / \mathrm{h}$

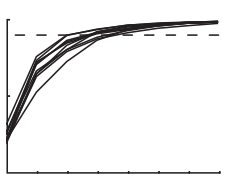

Tempora

Patterns

Pattern 1
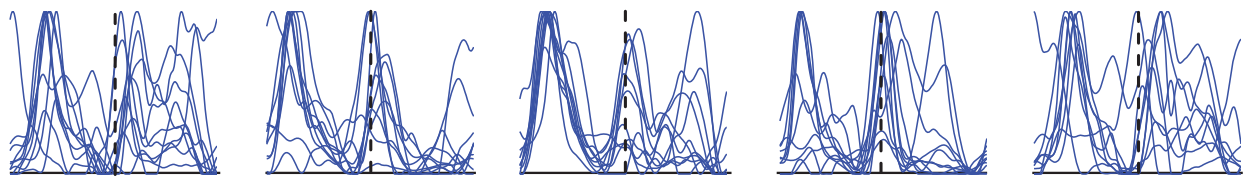

Pattern 2
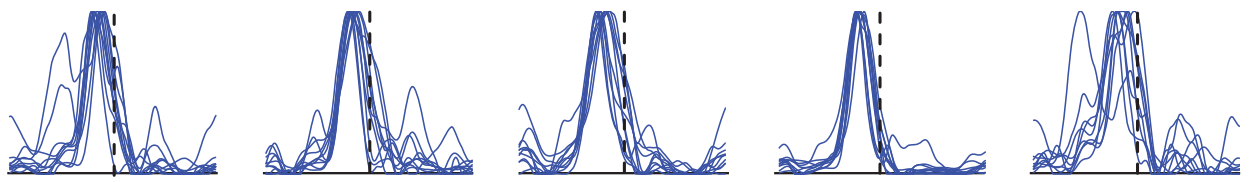

Pattern 3
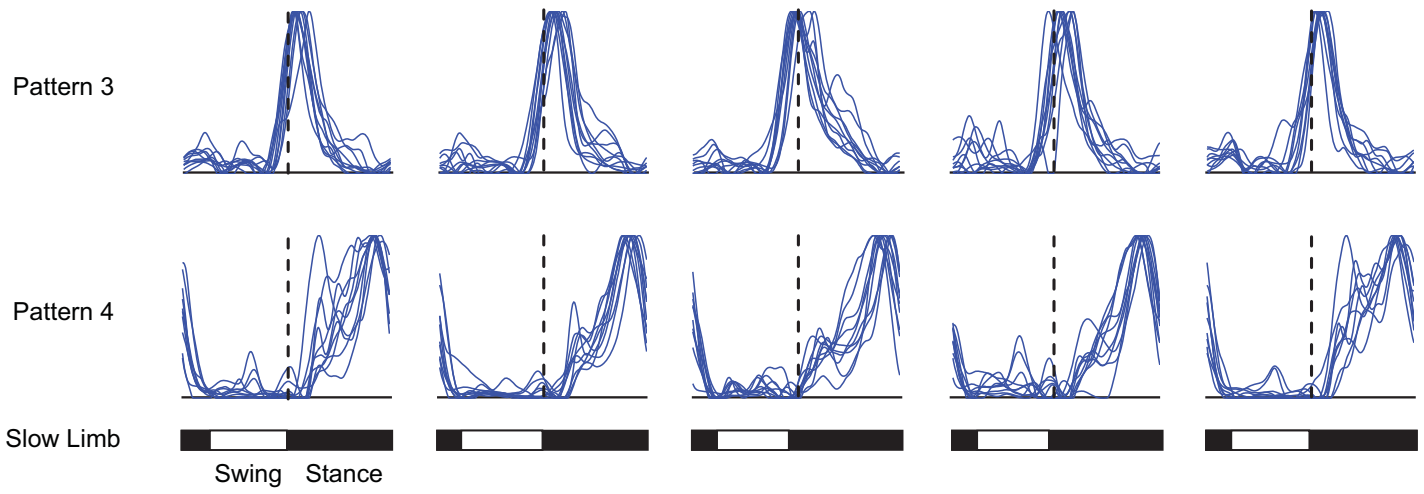

Muscle
Loadings

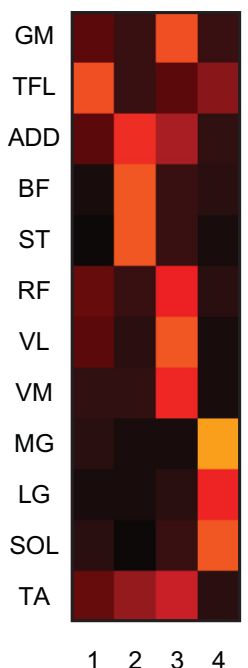

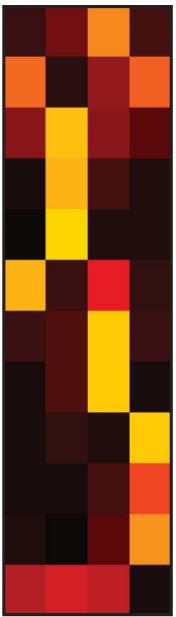

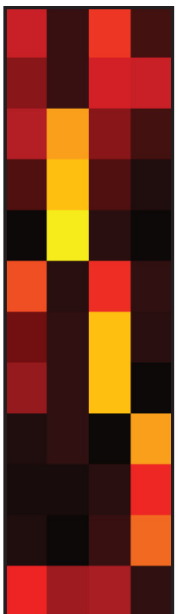

Pattern (\#)
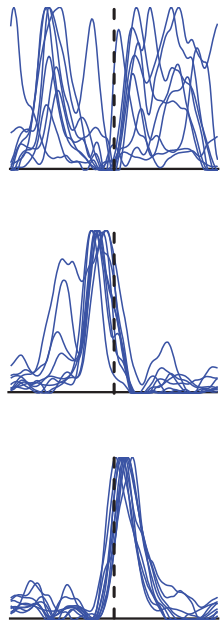

Late Post

Asymmerty

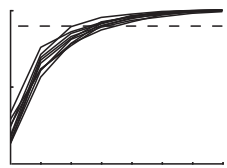

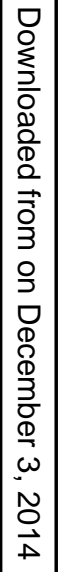
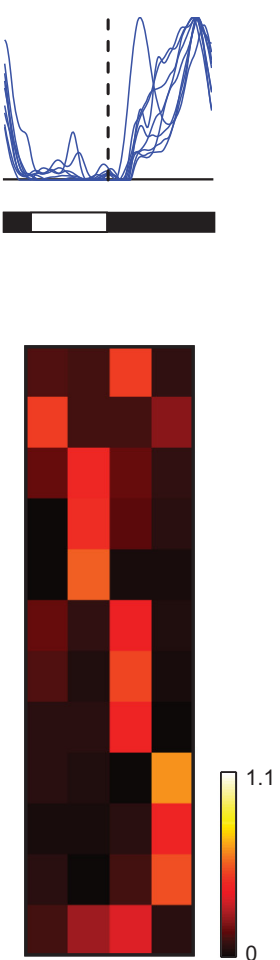

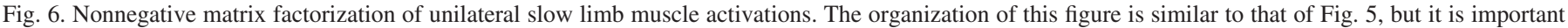

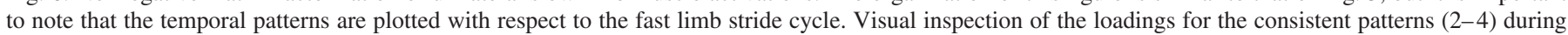
the split-belt conditions suggest their magnitudes are located between the 4 and $6 \mathrm{~km} / \mathrm{h}$ tied conditions, yet the limb is moving at $4 \mathrm{~km} / \mathrm{h}$. 

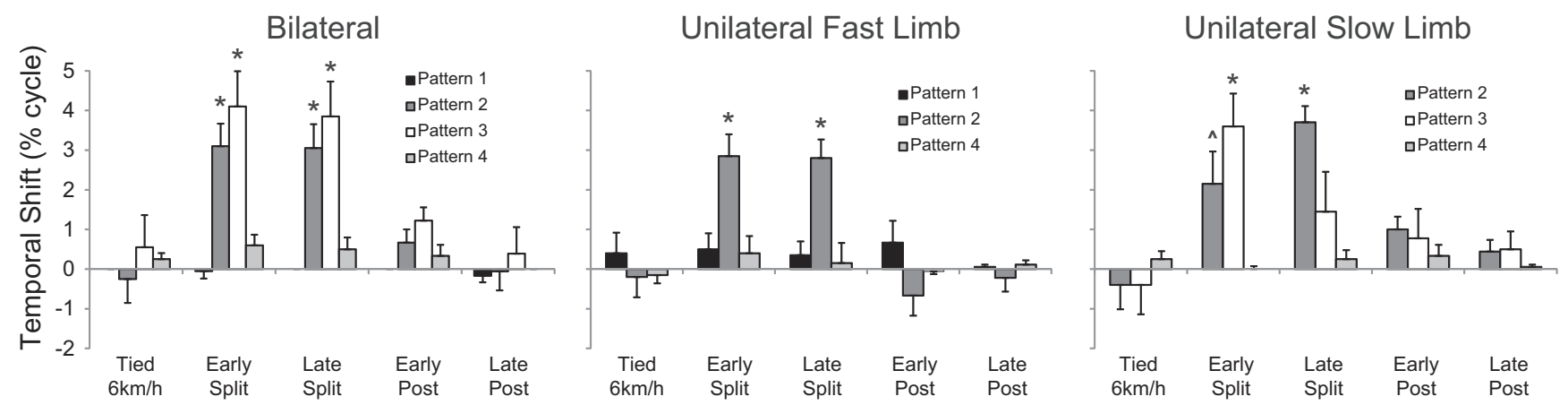

B

Kinematic Temporal Modulation
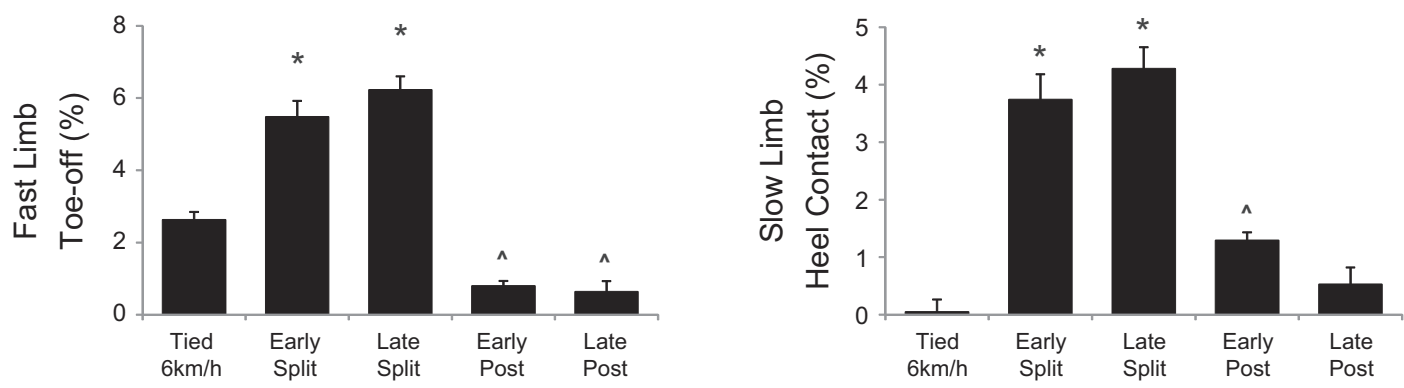

C

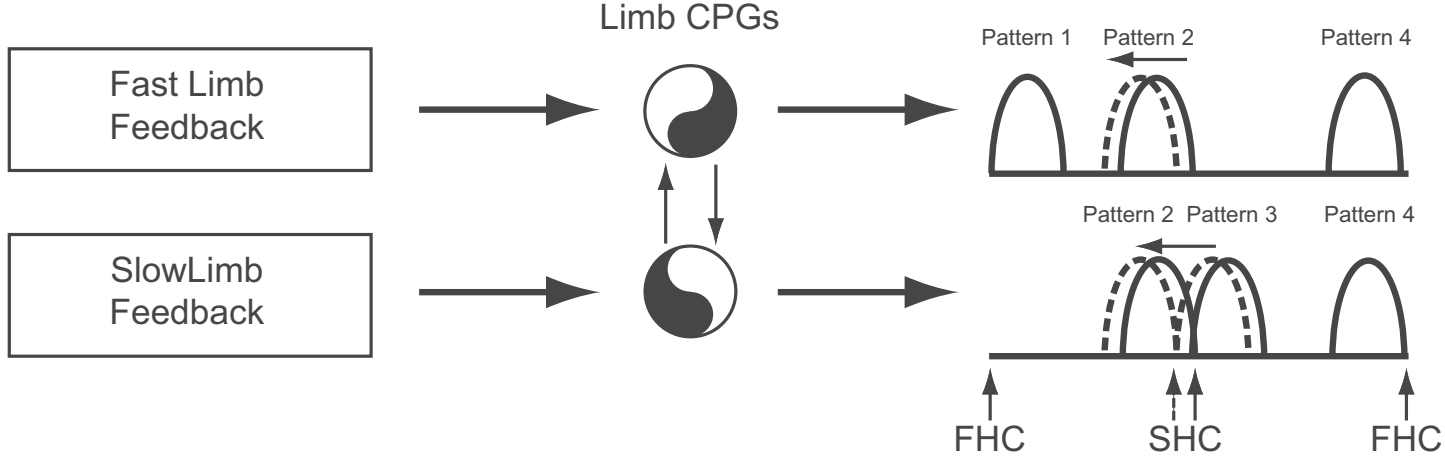

Fig. 7. Temporal modulation of variables for different treadmill conditions. Modulation of temporal patterns is shown in $A$, with a positive magnitude indicating a temporal shift to earlier in the gait cycle. In the unilateral plots, only the patterns that were consistent between participants are shown. $B$ shows the temporal modulation of fast limb toe-off (left) and slow limb heel contact (right) for the differing conditions. A value of " 0 " in these plots indicates no difference compared with the $4 \mathrm{~km} / \mathrm{h}$ condition. Positive values indicate the event is occurring earlier in the gait cycle. In $A$ and $B$, significant differences $(P<0.05)$ from all tied-belt conditions are indicated with an asterisk, and differences $(P<0.05)$ from only the $6 \mathrm{~km} / \mathrm{h}$ tied-belt condition are indicated with a caret. A proposed mechanism of central pattern generator (CPG) control is shown in $C$. Here, proprioceptive feedback is obtained from the fast and slow limbs, which is sent to limb CPGs. A tight coupling between each of the CPGs produces a coordinated output whereby shifting of individualized limb temporal patterns occurs simultaneously. Fast (FHC) and slow (SCH) limb heel contact are annotated, and pattern locations during tied (solid) and split-belt (dotted) conditions are shown.

work examining joint moments (Roemmich et al. 2012) and muscle activity contributions to center-of-mass acceleration (Jansen et al. 2013) during split-belt locomotion. With visual inspection of Fig. 2, one can see similar changes in gastrocnemius and tibialis anterior activity. Dietz et al. (1994) did note a time shift of gastrocnemius activity to the earlier part of the gait cycle in the faster limb during split-belt locomotion, but this was neither quantified nor expanded on in the context of parallel changes in the bilateral limb coordination. Finley et al. (2013) examined the changes in iEMG magnitude in four lower limb muscles during adaptations of locomotion on a split-belt treadmill. They showed a general decrease between the early and late stages of split-belt walking. Our results with an increased set of muscles agree with their findings by also showing a similar decrease between the early and late stages of the postasymmetric condition. By extrapolating the findings of Finley et al. (2013) to the current study, one may hypothesize that energy would be saved following a bout on a split-belt treadmill. 
Basic activation patterns. The current study focuses mainly on identifying basic underlying patterns in EMG activity and quantifying temporal adjustments of these patterns to the splitbelt conditions. Previous work has suggested that 4-5 basic patterns can account for $\sim 90 \%$ of the variance in locomotor EMG activity, although the estimated number of patterns depends on the number and locations of the recorded muscles (Ivanenko et al. 2004; McGowan et al. 2010; Monaco et al. 2010; Olree and Vaughan 1995). It is thought that these patterns or "primitives" are present at birth and are refined through development into adulthood (Dominici et al. 2011). The present study examined four such patterns in each condition, although only the temporal structures of three of these patterns were consistent between participants in the unilateral analyses. The fact that people retain these basic patterns during asymmetric locomotion (Figs. 4-6) illustrates their general robustness when adapting to environmental constraints.

The results reported here show a shift to an earlier time in the gait cycle for a select number of these patterns. Both bilateral and unilateral EMG analyses showed similar results, summarized in Fig. 7. In the bilateral analysis, the patterns associated with fast limb ankle plantar flexion/slow limb hamstring activity (pattern 2 in Fig. $4 B$ ) and slow limb quadriceps activity (pattern 3 in Fig. 4B) occurred earlier during the split-belt condition. These temporal shifts were confirmed in the unilateral analyses (fast limb plantar flexor, slow limb hamstring, and quadriceps). Examination of fast toe-off and slow limb heel contact (Fig. 7B) may suggest that these shifts are related to the asymmetric walking style generated by the split-belt treadmill. Earlier plantar flexion occurs to facilitate the advanced push-off in the fast limb, whereas earlier hamstring and quadriceps activity decelerate the swinging slow limb and accept the weight of the body, respectively, for advanced slow limb heel contact. These data are in line with a recent paper of Ogawa et al. (2014), which demonstrates that the anterior component of the braking force showed a clear pattern of adaptation. On the other hand, the findings also confirm the hypothesis that temporal shifting of locomotor patterns is tightly related to toe-off, as shown in Ivanenko et al. (2004) for the effect of walking speed. Moreover, similar suggestions are made during the study of limping, whereby the switch from stance to swing phase is linked to contralateral rather than ipsilateral limb mechanisms (De Visser et al. 2005).

It has long been argued that locomotion is controlled by spinal CPGs (Grillner 1979). Although the existence of CPG in humans is still debated, the basic locomotor patterns extracted from EMG data may be considered representative of hypothetical CPG output (Dominici et al. 2011; Ivanenko et al. 2003, 2004, 2006). The current results show a temporal modulation of the basic patterns when walking on a split-belt treadmill, and the mechanism for this is most likely related to proprioceptive feedback associated with these asymmetric conditions. As remarked above, past work has shown the importance of limb position, velocity, and loading when adapting locomotor patterns on a split-belt treadmill, and this afferent information is thought to influence CPG output (Dietz et al. 1994; Jensen et al. 1998; Prokop et al. 1995). Furthermore, Frigon et al. (2013) have shown that bilateral kinematic phase variations of cat hindlimbs are preserved following spinal cord transection, suggesting that this temporal organization between sides is located within the spinal cord, at least in this animal species. Here, we present a possible mechanism for hypothetical CPG modulation when walking on a split-belt treadmill (Fig. 7C). As stated, limb-specific proprioceptive input and supraspinal signals provide information to hypothetical limb-specific CPGs regarding the motion of the separate treadmill belts during asymmetric locomotion. These individual CPGs could then modulate the timings of basic locomotor patterns to generate a stable walking style in the altered environment. Such temporal output has been suggested to be a priority during locomotor adaptations (Malone et al. 2012). Moreover, the importance of bilateral communication between these limb-specific CPGs is illustrated here since the patterns related to fast limb plantar flexor activity and slow limb hamstring activity maintain their simultaneous bursts in all of the conditions examined in our study. This may be a possible mechanism of the underlying locomotor control studied here, but it should be remarked that this remains speculative given the lack of direct neurophysiological evidence for the existence and organization of CPG in humans.

It should be noted that our analysis of basic muscle patterns was not sensitive enough to detect any statistical differences between early/late split-belt and postasymmetry conditions, differences that are instead detectable from kinematic variables (Reisman et al. 2005). A fine-tuning of the neural control when adapting the locomotor patterns may be masked due to the small size of the effects (temporal shifts $\sim 3-4 \%$ of gait cycle; Fig. $7 A$ ) or be present in the residual $10-15 \%$ of the variance that was not explained by the patterns extracted by NNMF that we retained for further quantitative analyses.

In conclusion, we argued that proprioceptive information from the lower limbs modulates motor output with a tight bilateral organization during split-belt locomotion. This mechanism could potentially allow for bilateral locomotor adaptations in various environmental conditions during both normal and pathological gait, but future work is necessary to explore these conditions.

\section{GRANTS}

M. J. MacLellan, Y. P. Ivanenko, and F. Lacquaniti were supported by the Italian Ministry of Health, Italian Ministry of Education, University, and Research (PRIN Grant), Italian Space Agency (DCMC, CRUSOE, and COREA Grants) and European Union FP7-ICT Program (AMARSi Grant no. 248311). F. Massaad was supported by a Postdoctoral Fellowship of the Research Foundation - Flanders (FWO), a Krediet aan Navorsers from the FWO (1.5.129.13N), an F+ Fellowship from KU Leuven, and a scientific prize of the Foundation Van Goethem-Brichant, Belgium. S. M. Bruijn was supported by a grant from the Netherlands Organization for Scientific Research (NWO no. 451-12-041) and an FWO Grant (G.0901.11) to J. Duysens. J. Duysens has been funded by the Interuniversity Attraction Poles Program initiated by the Belgian Science Policy Office (P7/11).

\section{DISCLOSURES}

No conflicts of interest, financial or otherwise, are declared by the author(s).

\section{AUTHOR CONTRIBUTIONS}

M.J.M., Y.P.I., F.M., S.M.B., J.D., and F.L. conception and design of research; M.J.M., Y.P.I., F.M., S.M.B., J.D., and F.L. performed experiments; M.J.M. and Y.P.I. analyzed data; M.J.M., Y.P.I., F.M., S.M.B., J.D., and F.L. interpreted results of experiments; M.J.M. and Y.P.I. prepared figures; M.J.M., Y.P.I., F.M., S.M.B., J.D., and F.L. drafted manuscript; M.J.M., Y.P.I., F.M., S.M.B., J.D., and F.L. edited and revised manuscript; M.J.M., Y.P.I., F.M., S.M.B., J.D., and F.L. approved final version of manuscript. 


\section{REFERENCES}

Butt SJ, Kiehn O. Functional identification of interneurons responsible for left-right coordination of hindlimbs in mammals. Neuron 38: 953-963, 2003.

Choi JT, Bastian AJ. Adaptation reveals independent control networks for human walking. Nat Neurosci 10: 1055-1062, 2007.

d'Avella A, Saltiel P, Bizzi E. Combinations of muscle synergies in the construction of a natural motor behavior. Nat Neurosci 6: 300-308, 2003.

Davis BL, Vaughan CL. Phasic behavior of EMG signals during gait: use of multivariate statistics. J Electromyogr Kinesiol 3: 51-60, 1993.

De Visser E, Veth RP, Schreuder HW, Duysens J. Altered phase-transitions in tibialis anterior and medial gastrocnemius during walking after limb saving surgery. Clin Neurophysiol 116: 2741-2747, 2005.

Dietz V, Zijlstra W, Duysens J. Human neuronal interlimb coordination during split-belt locomotion. Exp Brain Res 101: 513-520, 1994.

Dominici N, Ivanenko YP, Cappellini G, d'Avella A, Mondì V, Cicchese M, Fabiano A, Silei T, Di Paolo A, Giannini C, Poppele RE, Lacquaniti F. Locomotor primitives in newborn babies and their development. Science 334: 997-999, 2011.

Elias LJ, Bryden MP, Bulman-Fleming MB. Footedness is a better predictor than is handedness of emotional lateralization. Neuropsychologia 36: $37-$ 43, 1998.

Finley JM, Bastian AJ, Gottschall JS. Learning to be economical: the energy cost of walking tracks motor adaptation. J Physiol 591: 1081-1095, 2013.

Forssberg H, Grillner S, Halbertsma J, Rossignol S. The locomotion of the low spinal cat. II. Interlimb coordination. Acta Physiol Scand 108: 283-295, 1980.

Frigon A, Hurteau MF, Thibaudier Y, Leblond H, Telonio A, D'Angelo G. Split-belt walking alters the relationship between locomotor phases and cycle duration across speeds in intact and chronic spinalized adult cats. $J$ Neurosci 33: 8559-8566, 2013.

Giszter SF, Hart CB. Motor primitives and synergies in the spinal cord and after injury-the current state of play. Ann NY Acad Sci 1279: 114-126, 2013.

Grillner S. Interaction between central and peripheral mechanisms in the control of locomotion. Prog Brain Res 50: 227-235, 1979.

Halbertsma JM. The stride cycle of the cat: the modelling of locomotion by computerized analysis of automatic recordings. Acta Physiol Scand Suppl 521: 1-75, 1983.

Hermens HJ, Freriks B, Disselhorst-Klug C, Rau G. Development of recommendations for SEMG sensors and sensor placement procedures. $J$ Electromyogr Kinesiol 10: 361-374, 2000.

Hoogkamer W, Bruijn SM, Duysens J. Stride length asymmetry in split-belt locomotion. Gait Posture 39: 652-654, 2014.

Ivanenko YP, Cappellini G, Dominici N, Poppele RE, Lacquaniti F. Coordination of locomotion with voluntary movements in humans. J Neurosci 25: 7238-7253, 2005.

Ivanenko Y, Grasso R, Zago M, Molinari M, Scivoletto G, Castellano V, Macellari V, Lacquaniti F. Temporal components of the motor patterns expressed by the human spinal cord reflect foot kinematics. J Neurophysiol 90: 3555-3565, 2003.

Ivanenko YP, Poppele RE, Lacquaniti F. Five basic muscle activation patterns account for muscle activity during human locomotion. $J$ Physiol 556: 267-282, 2004

Ivanenko YP, Poppele RE, Lacquaniti F. Motor control programs and walking. Neuroscientist 12: 339-348, 2006.

Jansen K, De Groote F, Duysens J, Jonkers I. Muscle contributions to center of mass acceleration adapt to asymmetric walking in healthy subjects. Gait Posture 38: 739-744, 2013.
Jensen L, Prokop T, Dietz V. Adaptational effects during human split-belt walking: influence of afferent input. Exp Brain Res 118: 126-130, 1998.

Kiehn O. Locomotor circuits in the mammalian spinal cord. Annu Rev Neurosci 29: 279-306, 2006.

Lacquaniti F, Ivanenko YP, Zago M. Patterned control of human locomotion. J Physiol 590: 2189-2199, 2012.

Malone LA, Bastian AJ, Torres-Oviedo G. How does the motor system correct for errors in time and space during locomotor adaptation? J Neurophysiol 108: 672-683, 2012.

McCrea DA, Rybak IA. Organization of mammalian locomotor rhythm and pattern generation. Brain Res Rev 57: 134-146, 2008.

McGowan CP, Neptune RR, Clark DJ, Kautz SA. Modular control of human walking: adaptations to altered mechanical demands. J Biomech 43: 412-419, 2010.

Monaco V, Ghionzoli A, Micera S. Age-related modifications of muscle synergies and spinal cord activity during locomotion. J Neurophysiol 104: 2092-2102, 2010.

Morton SM, Bastian AJ. Cerebellar contributions to locomotor adaptations during splitbelt treadmill walking. J Neurosci 26: 9107-9116, 2006.

Nanhoe-Mahabier W, Snijders AH, Delval A, Weerdesteyn V, Duysens J, Overeem S, Bloem BR. Split-belt locomotion in Parkinson's disease with and without freezing of gait. Neuroscience 236: 110-116, 2013.

Neptune RR, Clark DJ, Kautz SA. Modular control of human walking: a simulation study. J Biomech 42: 1282-1287, 2009.

Ogawa T, Kawashima N, Ogata T, Nakazawa K. Predictive control of ankle stiffness at heel contact is a key element of locomotor adaptation during split-belt treadmill walking in humans. J Neurophysiol 111: 722-732, 2014.

Olree KS, Vaughan CL. Fundamental patterns of bilateral muscle activity in human locomotion. Biol Cybern 73: 409-414, 1995.

Patla AE. Some characteristics of EMG patterns during locomotion: implications for the locomotor control process. J Mot Behav 17: 443-461, 1985.

Prokop T, Berger W, Zijlstra W, Dietz V. Adaptational and learning processes during human split-belt locomotion: interaction between central mechanisms and afferent input. Exp Brain Res 106: 449-456, 1995.

Reisman DS, Block HJ, Bastian AJ. Interlimb coordination during locomotion: what can be adapted and stored? J Neurophysiol 94: 2403-2415, 2005.

Reisman DS, Wityk R, Silver K, Bastian AJ. Locomotor adaptation on a split-belt treadmill can improve walking symmetry post-stroke. Brain 130: 1861-1872, 2007.

Roemmich RT, Stegemöller EL, Hass CJ. Lower extremity sagittal joint moment production during split-belt treadmill walking. J Biomech 45: 2817-2821, 2012.

Schneiders AG, Sullivan SJ, O’Malley KJ, Clarke SV, Knappstein SA, Taylor LJ. A valid and reliable clinical determination of footedness. $P M R$ 2: 835-841, 2010.

Thelen E, Ulrich BD, Niles D. Bilateral coordination in human infants: stepping on a split-belt treadmill. J Exp Psychol Hum Percept Perform 13: 405-410, 1987.

Ting LH, Chvatal SA, Safavynia SA, McKay JL. Review and perspective: neuromechanical considerations for predicting muscle activation patterns for movement. Int J Numer Method Biomed Eng 28: 1003-1014, 2012.

Tresch MC, Cheung VC, d'Avella A. Matrix factorization algorithms for the identification of muscle synergies: evaluation on simulated and experimental data sets. J Neurophysiol 95: 2199-2212, 2006.

Yang JF, Lamont EV, Pang MY. Split-belt treadmill stepping in infants suggests autonomous pattern generators for the left and right leg in humans. J Neurosci 25: 6869-6876, 2005. 